
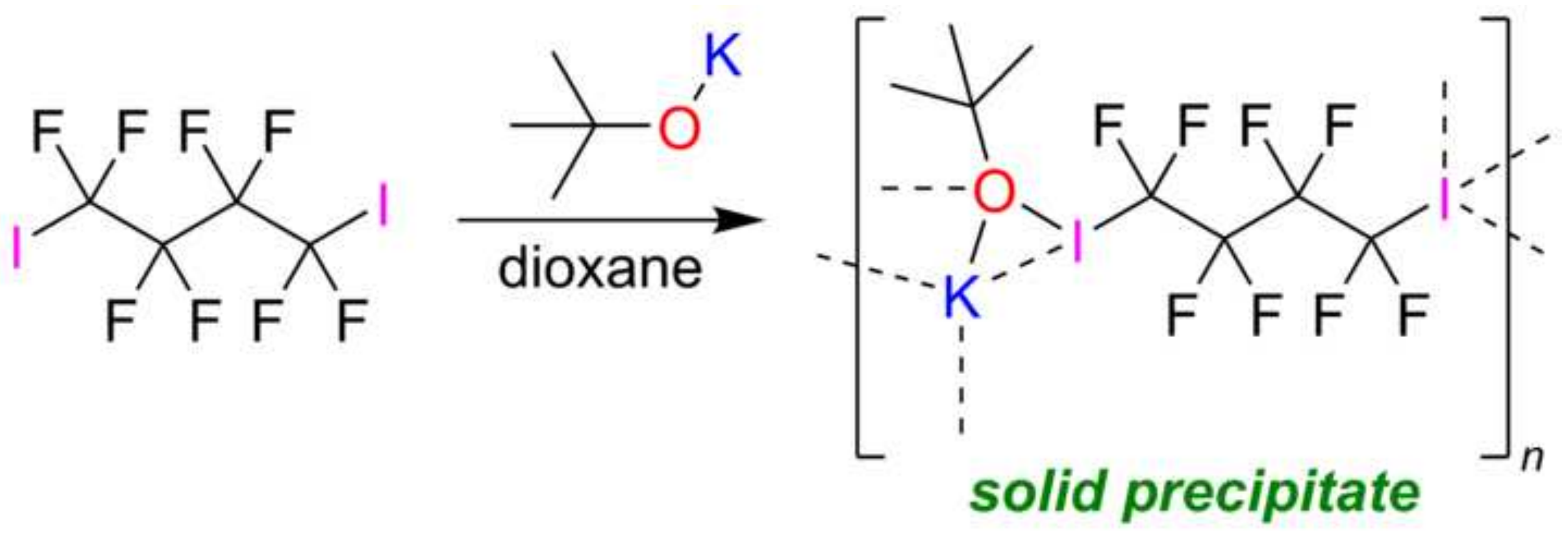


\section{Graphical Abstract - Synopsis}

1,4-diiodooctafluorobutane was found to react with potassium tert-butoxide in dioxane to afford a halogen-bonded adduct as a white precipitate. Single crystal X-ray analysis of the precipitate reveals an extended network held together by strong iodine-oxygen interactions. The oxygen-iodine bond length in the tert-butoxide complex was found to be $2.391(6) \AA$, much shorter than a previously published and similar complex involving dioxane (2.814(12) Å). To our knowledge, this work represents the first example of a structurally characterized halogen bonding network involving anionic oxygen donor atoms with perfluoroalkyl iodides. The tert-butoxide adduct displays the shortest iodine-oxygen distance and the longest iodine-carbon distance thus far reported for any halogen bonding involving perfluoroalkyl iodide substrate. 


\title{
An Unusual Example of Halogen Bonding to Potassium t-Butoxide
}

\author{
Long $\mathrm{Xu}^{\dagger}{ }^{\dagger}$ Roger E. Cramer, ${ }^{\S}$ and David A. Vicic ${ }^{+*}$
}

${ }^{\dagger}$ Department of Chemistry, Lehigh University, 6 E. Packer Ave., Bethlehem, PA 18015. E-mail:

vicic@lehigh.edu; Phone: 1-610-758-3466; Fax: 1-610-758-6536, and ${ }^{\S}$ the Department of Chemistry, University of Hawaii, 2545 McCarthy Mall, Honolulu, HI 96822

Table of Contents Graphic

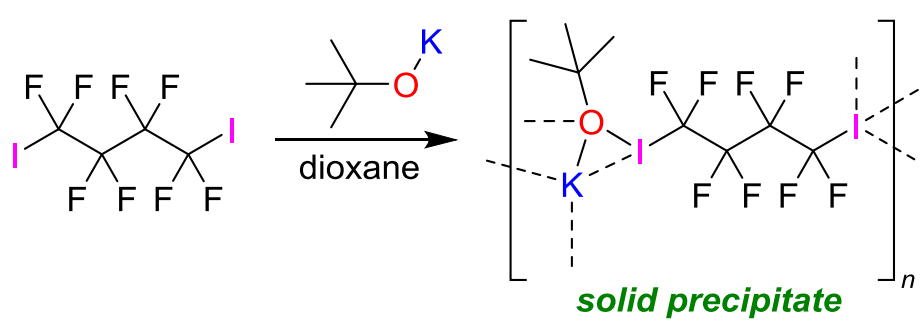

Abstract: 1,4-diiodooctafluorobutane was found to react with potassium tert-butoxide in dioxane to afford a halogen-bonded adduct as a white precipitate. Single crystal X-ray analysis of the precipitate reveals an extended network held together by strong iodine-oxygen interactions. The oxygen-iodine bond length in the tert-butoxide complex was found to be $2.391(6) \AA$, much shorter than a previously published and similar complex involving dioxane (2.814(12) $\AA$ ). To our knowledge, this work represents the first example of a structurally characterized halogen bonding network involving anionic oxygen donor atoms with perfluoroalkyl iodides. The tert-butoxide adduct displays the shortest iodine-oxygen distance and the longest iodine-carbon distance thus far reported for any halogen bonding involving a perfluoroalkyl iodide substrate.

Keywords: Fluoroalkylation; halogen bonding, fluorine 


\section{Introduction}

The ability of halogen atoms in certain molecules to interact with electron donors has been exploited in such fields as crystal engineering, anion recognition, and self-assembly processes.[1-9] Halogen bonding may also be important for mechanistic considerations in synthetic reactions, [10] and as complements or alternatives to hydrogen bonding in the assembly of biological supermolecules.[11] According to IUPAC, "A halogen bond occurs when there is evidence of a net attractive interaction between an electrophilic region associated with a halogen atom in a molecular entity and a nucleophilic region in another, or the same, molecular entity." [12]

Perfluoroalkyl iodides have served as an especially active halogen bonding partners, especially to nitrogen donor molecules.[13-17] X-ray analyses of these nitrogen supported halogen-bonded architectures indicate that the $\mathrm{N} \cdots$. bond length is typically around $2.80 \AA$ in the solid state (or approximately 0.8 times the sum of the van der Waals radii for nitrogen and iodine).[16] Moreover, the angle formed by the covalent and the noncovalent bondings around the halogen were approximately linear (spanning from $177^{\circ}$ to $165^{\circ}$ ), consistent with the $n \rightarrow \sigma^{*}$ character of the interaction. $[18,19]$ Furthermore, it was observed that in general, the stronger the halogen bonding, the greater the lengthening of the C-I bond, consistent with an electron donation from nitrogen to iodine.[20] Resnati and co-workers showed that when $\alpha, \omega$-perfluoroalkyl diiodides were used as a substrate, the donoracceptor interactions involving nitrogen-iodine bonds are doubled, affording strongly bound selfassembled and infinite one-dimensional chains.[16] Indeed, upon reaction of perfluoroalkyl polyiodides with neutral bidentate nitrogen donor molecules, supramolecular architectures of various flavors could be obtained. $[3,16]$ 


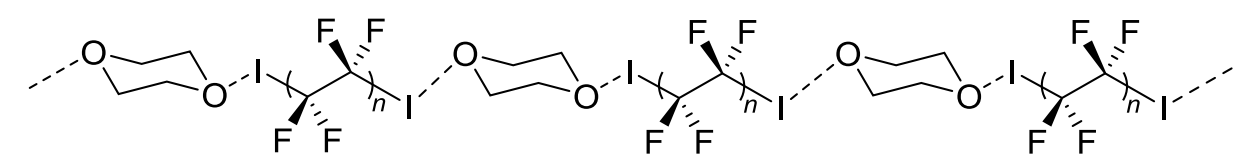

Figure 1. Infinite chains formed by oxygen-iodine interactions of $\mathbf{1}(n=1)$ in the solid state.[17]

Oxygen is a weaker electron donor than nitrogen for halogen bonding,[17] and so thus far the majority of examples of halogen bonding to $\alpha, \omega$-perfluoroalkyl diiodides have been with nitrogen donors like amines and imines. Zhu and co-workers, however, showed crystallographically that oxygen donors like dioxane and hexamethylphosphoramide can form infinite chains with $\alpha, \omega$-perfluoroalkyl diiodides like that shown in Figure 1.[17] The distance between oxygen and iodine in 1 was measured to be 2.814(12) $\AA$, which was shorter than the sum of the van der Waals radii of iodine (1.98 $\AA$ ) and oxygen $(1.52 \AA)$. This example showed clearly the nature and the degree of the halogen bonding with neutral oxygen donors molecules. However, there have been no examples of halogen bonding of perfluoroalkyl iodides with anionic oxygen donor molecules that have been structurally characterized. Such an adduct would be interesting to analyze, as although amines with higher energy lone pairs form more stable adducts than do neutral oxygen donors, anionic oxygen donors may have the energy of its lone pair raised relative to amines leading to even stronger halogen bonded adducts. An infrared and NMR study has detected a halogen bonding interaction between $\mathrm{CF}_{3}$ l and $\left[\mathrm{Me}_{4} \mathrm{~N}\right]\left[\mathrm{OCF}_{3}\right]$, but that species was stable only at low temperatures.[13] Herein, we describe in detail the strong and stable halogen bond that forms between 1,4-diiodooctafluorobutane and potassium tert-butoxide.

\section{Results and Discussion}

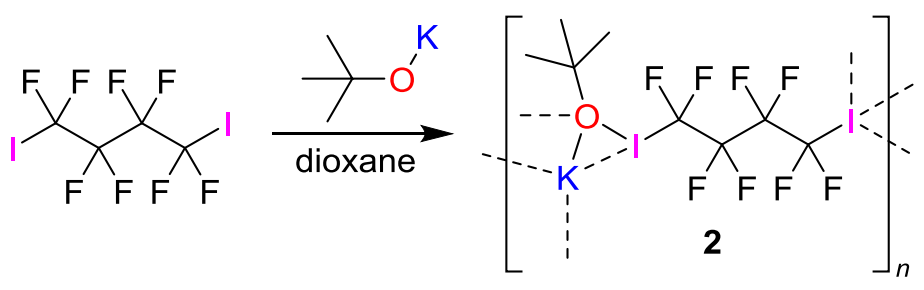


Upon mixing 1,4-diiodooctafluorobutane with potassium tert-butoxide in dioxane, the immediate precipitation of $\mathbf{2}$ as a white solid (eq 1 ) was observed. Solvent choice was critical as the use of acetonitrile instantly generated $\mathrm{H}\left(\mathrm{CF}_{2}\right)_{4} \mathrm{H}$ and led to a black mixture at room temperature. The formation of $\mathrm{H}\left(\mathrm{CF}_{2}\right)_{4} \mathrm{H}$ was also observed in $\mathrm{THF}, \mathrm{Et}_{2} \mathrm{O}$ and DMSO. The compound was stable in DMF and benzonitrile, however, and showed no signs of $\mathrm{H}\left(\mathrm{CF}_{2}\right)_{4} \mathrm{H}$ formation in those solvents. NMR spectroscopy supported the formation of a halogen-bonded adduct. The ${ }^{19} \mathrm{~F}$ NMR chemical shifts for the pure 1,4diiodooctafluorobutane in DMF appear at $\delta=-61$ and -113 , whereas the halogen bonded complex 2 exhibited resonances far more upfield at $\delta=-88$ and -114 in DMF. A strong binding interaction with potassium tert-butoxide is not unique to the 1,4-diiodooctafluorobutane substrate. If the monoiodide species 1-iodo-perfluorobutane is used as the acceptor, an even larger $\Delta \delta=26$ for the $\mathrm{RCF}_{2}$ I signals was observed. However, unlike 1,4-diiodooctafluorobutane, the potassium tert-butoxide complex with 1iodo-perfluorobutane does not precipitate from dioxane, suggesting a supramolecular lattice similar to 2 is not formed. ${ }^{19} \mathrm{~F}$ NMR chemical shift differences have previously been used to characterize the strength of the halogen bonding. It was established that $\mathrm{RCF}_{2} \mathrm{X}$ signals show large high-field shifts upon halogen bonding formation, and the stronger the interaction, the larger the shift.[19] The $\Delta \delta=21$ and 26 in DMF solvent for the $\mathrm{CF}_{2} \mathrm{I}$ resonances that we observed for the tert-butoxide adducts are the largest differences thus far reported for halogen bonding interactions involving perfluoroalkyl iodides. For comparison, $\Delta \delta=4.9$ was observed for the neutral dioxane adduct 1.[17]

Since we were unaware of any examples of halogen bonding of perfluoroalkyl iodides to potassium tertbutoxide, an X-ray investigation of $\mathbf{2}$ was performed. The solid that precipitated from dioxane could be recrystallized from DMF/trifluorotoluene, and the X-ray analysis confirms the nature of the bonding in $\mathbf{2}$ (Figure 2). While the iodine-oxygen bond length in the dioxane complex 1 was measured to be $2.814(12) \AA$, [17] the iodine-oxygen bond length in $\mathbf{2}$ was found to be much shorter at $2.391(6) \AA$. This distance approaches the average iodine-oxygen covalent bond of $(2.14 \AA)$ ).[17, 21] The iodine-carbon 
bond length in $\mathbf{2}$ is $2.281(8) \AA$, which to our knowledge is the largest elongation observed in halogenbonded complexes to perfluoroalkyl iodides and is consistent with an electron donation from oxygen to iodine.

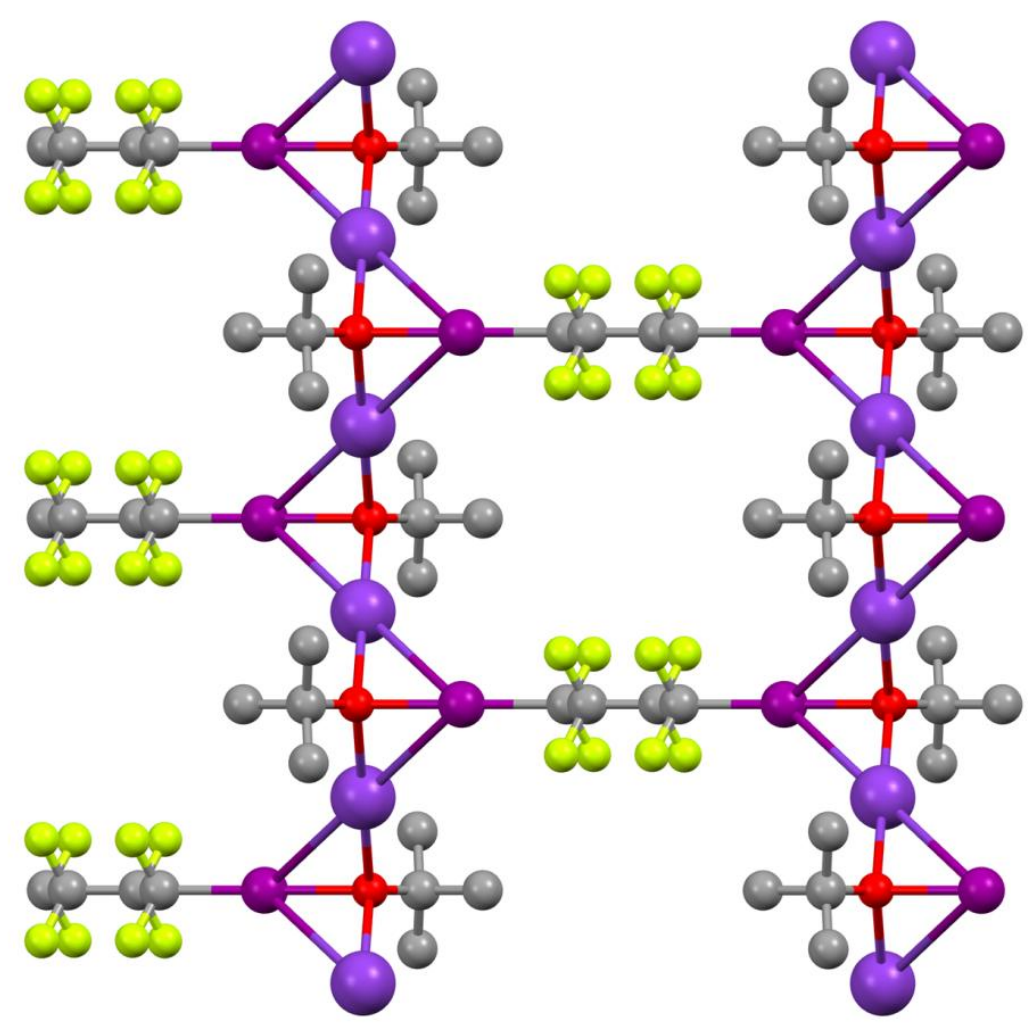

Figure 2. Solid-state structure depicting the networked arrangement of 2. Color scheme: O red, C grey, F yellow-green, I magenta, $\mathrm{K}$ purple. The disordered potassium-bound DMF solvent molecules have been omitted for clarity. Selected bond lengths ( $\AA$ ): iodine-carbon 2.281(8); iodine-oxygen 2.391(6).

With regards to the supramolecular architecture that is produced, the X-ray analysis reveals that the use of anionic instead of neutral oxygen donors has introduced another dimensionality to the extended molecular network. The network is not an infinite chain, as has previously been observed with neutral oxygen donors, but rather infinite sheets by virtue of the fact that the potassium counter-ions can chelate to multiple iodine atoms. This data raises the intriguing possibility that the use of other countercations might lead to more complex architectures, including those possessing three-dimensionality. 


\section{Conclusions}

We have shown that 1,4-diiodooctafluorobutane reacts with potassium tert-butoxide in dioxane to afford a halogen-bonded adduct with an extended two-dimensional network held together by strong iodine-oxygen interactions. The new complex represents the first example of a structurally characterized halogen bonding network involving anionic oxygen donor atoms with perfluoroalkyl iodides. The tert-butoxide adduct displays the shortest iodine-oxygen distance and the longest iodinecarbon distance thus far reported for any halogen bonding interactions involving perfluoroalkyl iodide substrate.

\subsection{Experimental Procedures}

4.1 General Considerations: All manipulations were performed under an inert atmosphere using standard Schlenk and high vacuum techniques or in a nitrogen filled glovebox. ${ }^{11}$ Solvents were purified by passing through activated alumina and/or copper in a solvent purification system supplied by Pure Process Technology. 1,4-diiodooctafluorobutane was purchased from SynQuest Labs, Inc. and used without further purification. Solution ${ }^{1} \mathrm{H}$ NMR spectra were recorded at ambient temperature on a Bruker DRX $500 \mathrm{MHz}$ spectrometer and referenced to residual proton solvent signals. ${ }^{13} \mathrm{C}$ NMR spectra were recorded on a Bruker NMR spectrometer operating at $125 \mathrm{MHz}$ and referenced to solvent signals.

${ }^{19} \mathrm{~F}$ spectra were recorded on the Bruker NMR spectrometer operating at $470 \mathrm{MHz}$ and referenced to $\alpha, \alpha, \alpha$-trifluorotoluene at $\delta$-67.3. A Bruker D8 Quest diffractometer was used for X-ray crystal structure determinations.

4.1.1. Synthesis of complex 2: $450 \mathrm{mg}(4 \mathrm{mmol})$ of potassium $t$-butoxide was dissolved in $4 \mathrm{~mL}$ of $1,4-$ dioxane. To this was added dropwise $453 \mathrm{mg}(1 \mathrm{mmol})$ of 1,4-diiodooctafluorobutane in $4 \mathrm{~mL}$ of 1,4dioxane. The mixture was stirred at room temperature for $15 \mathrm{~min}$. Then the mixture was filtered through a glass frit and the residue was washed with diethyl ether. The residue was then dried under 
vacuum to remove all the volatiles to yield $780 \mathrm{mg}$ of an off-white powder (the amount of dioxane molecules coordinated to potassium at this stage is unknown). The off-white powder was then dissolved into a 1:5 mixture of DMF:trifluorotoluene and placed in a $-35^{\circ} \mathrm{C}$ freezer overnight to form colorless crystals suitable for X-ray analysis. $\left.{ }^{19} \mathrm{~F} \mathrm{NMR} \mathrm{(DMF-} d_{7}, 470 \mathrm{MHz}\right): \delta-88$ (br s), -114 (br s). The thermal and air-sensitivity of $\mathbf{2}$ precluded elemental analysis. Crystallographic data (excluding structure factors) for compound $\mathbf{2}$ has been deposited with the Cambridge Crystallographic Data Centre as supplementary publication number CCDC 1050288. Copies of the data can be obtained free of charge on application to CCDC, 12 Union Road, Cambridge CB2 1EZ, UK [fax: +44 1223336 033; e-mail: deposit@ccdc.cam.ac.uk].

Acknowledgements: D.A.V. thanks the Office of Basic Energy Sciences of the U.S. Department of Energy (DE-FG02-13ER16369) for support of this work.

\section{References}

[1] M. Erdelyi, Halogen bonding in solution, Chem. Soc. Rev., 41 (2012) 3547-3557.

[2] A.C. Legon, The halogen bond: an interim perspective, Phys. Chem. Chem. Phys., 12 (2010) 77367747.

[3] P. Metrangolo, F. Meyer, T. Pilati, G. Resnati, G. Terraneo, Halogen bonding in supramolecular chemistry, Angew. Chem., Int. Ed., 47 (2008) 6114-6127.

[4] P. Metrangolo, G. Resnati, Halogen Bonding: Where We Are and Where We Are Going, Cryst. Growth Des., 12 (2012) 5835-5838.

[5] P. Metrangolo, G. Resnati, Metal-bound halogen atoms in crystal engineering, Chem. Commun., 49 (2013) 1783-1785.

[6] A. Mukherjee, S. Tothadi, G.R. Desiraju, Halogen Bonds in Crystal Engineering: Like Hydrogen Bonds yet Different, Acc. Chem. Res., 47 (2014) 2514-2524.

[7] P. Politzer, P. Lane, M.C. Concha, Y. Ma, J.S. Murray, An overview of halogen bonding, J. Mol. Model., 13 (2007) 305-311.

[8] P. Politzer, J.S. Murray, T. Clark, Halogen bonding: an electrostatically-driven highly directional noncovalent interaction, Phys. Chem. Chem. Phys., 12 (2010) 7748-7757.

[9] A. Priimagi, G. Cavallo, P. Metrangolo, G. Resnati, The Halogen Bond in the Design of Functional Supramolecular Materials: Recent Advances, Acc. Chem. Res., 46 (2013) 2686-2695.

[10] N.S. Zefirov, D.I. Makhon'kov, X-philic reactions, Chem. Rev., 82 (1982) 615-624.

[11] P. Metrangolo, G. Resnati, Halogen versus Hydrogen, Science, 321 (2008) 918-919. 
[12] G.R. Desiraju, P.S. Ho, L. Kloo, A.C. Legon, R. Marquardt, P.P. Metrangolo, G. Resnati, K. Rissanen, Definition of the halogen bond (IUPAC Recommendations 2013), Pure Appl. Chem., 85 (2013) 1711-1713.

[13] R. Minkwitz, R. Broechler, M. Berkei, Preparation of trifluoromethylhalogen iodate(I) salts $\left(\mathrm{CH}_{3}\right)_{4} \mathrm{~N}^{+} \mathrm{CF}_{3} \mathrm{IX}-(\mathrm{X}=\mathrm{F}, \mathrm{Cl}, \mathrm{Br})$ and trifluoromethyltrifluoromethoxy iodate(I) $\left(\mathrm{CH}_{3}\right)_{4} \mathrm{~N}^{+} \mathrm{CF}_{3} \mathrm{OOCF}_{3}^{-}$ salts, Z. Anorg. Allg. Chem., 622 (1996) 1749-1755.

[14] R. Minkwitz, T. Hertel, A. Braeutigam, Formation and spectroscopic characterization of tetramethylammonium trifluoromethylnitratoiodate(I) $\left(\mathrm{CH}_{3}\right)_{4} \mathrm{~N}^{+} \mathrm{CF}_{3} \mid \mathrm{ONO}_{2}$, Z. Anorg. Allg. Chem., 623 (1997) 151-154.

[15] R. Minkwitz, J. Jakob, On the preparation of tetramethylammonium trifluoromethylbistrifluoromethylnitroxyliodate(I) $\left(\mathrm{CH}_{3}\right)_{4} \mathrm{~N}^{+}\left(\mathrm{CF}_{3}\right)_{2} \mathrm{NOICF}_{3}{ }^{-}$and the reaction of tetramethylammonium bistrifluoromethylnitrate $\left(\mathrm{CH}_{3}\right)_{4} \mathrm{~N}^{+}\left(\mathrm{CF}_{3}\right)_{2} \mathrm{NO}^{-}$with $\mathrm{Cl}_{2}$ and $\mathrm{ClONO}_{2}, \mathrm{Z}$. Anorg. Allg. Chem., 623 (1997) 613-618.

[16] P. Cardillo, E. Corradi, A. Lunghi, S. Valdo Meille, T.M. Messina, P. Metrangolo, G. Resnati, The $\mathrm{N}$ … Intermolecular Interaction as a General Protocol for the Formation of PerfluorocarbonHydrocarbon Supramolecular Architectures, Tetrahedron, 56 (2000) 5535-5550.

[17] Q. Chu, Z. Wang, Q. Huang, C. Yan, S. Zhu, Fluorine-Containing Donor-Acceptor Complex: Infinite Chain Formed by Oxygen ‥lodine Interaction, J. Am. Chem. Soc., 123 (2001) 11069-11070.

[18] P. Metrangolo, W. Panzeri, F. Recupero, G. Resnati, Perfluorocarbon-hydrocarbon self-assembly Part 16. ${ }^{19} \mathrm{~F}$ NMR study of the halogen bonding between halo-perfluorocarbons and heteroatom containing hydrocarbons, J. Fluorine Chem., 114 (2002) 27-33.

[19] P. Metrangolo, G. Resnati, Halogen bonding: a paradigm in supramolecular chemistry, Chem. Eur. J., 7 (2001) 2511-2519.

[20] A. Farina, S.V. Meille, M.T. Messina, P. Metrangolo, G. Resnati, G. Vecchio, Perfluorocarbonhydrocarbon self-assembly, part 5. Resolution of racemic 1,2-dibromohexafluoropropane through halogen-bonded supramolecular helices, Angew. Chem., Int. Ed., 38 (1999) 2433-2436.

[21] F.H. Allen, O. Kennard, D.G. Watson, L. Brammer, A.G. Orpen, R. Taylor, Tables of bond lengths determined by $\mathrm{X}$-ray and neutron diffraction. Part 1 . Bond lengths in organic compounds, Journal of the Chemical Society, Perkin Transactions 2, (1987) S1-S19. 